\title{
¿COHEREnCIA O RACIONALIDAD?
}

Manuel Atienza *

* Universidad de Alicante (España)
He discutido con Amalia Amaya sobre la coherencia durante muchos años: durante todos los que dedicó a elaborar su (magnífica) tesis de doctorado sobre ese tema. Por razones que no vienen al caso no estuve presente en la lectura de su tesis (era uno de los miembros del tribunal encargado de juzgarla), de manera que agradezco la oportunidad que me brinda Discusiones para hacer algún comentario sobre ese trabajo o, si se quiere, sobre las conclusiones principales que Amalia ha extraído de su investigación y que presenta aquí en la forma clara, sugerente y elegante que el lector podrá apreciar en seguida.

No voy a decir que Amalia lo sabe todo sobre la coherencia; como "todo", al menos en este contexto, es un 
concepto abierto y dinámico, es posible (por no decir casi seguro) que en las últimas semanas o meses se hayan publicado diversos trabajos sobre la coherencia que pueden haber aportado alguna nueva cosa que ella no conozca. Pero lo que sí me atrevo a decir (y a presentar como un enunciado verdadero, se suscriba o no una concepción "coherentista" de la verdad) es que Amalia Amaya es la persona que más sabe sobre la coherencia. Ha estudiado el tema desde todas las perspectivas -las disciplinas- relevantes y lo ha hecho de manera profunda, con una competencia asombrosa. Su tesis de doctorado (que en seguida se convertirá en libro) va a constituir, sin duda, un punto de referencia indispensable para todo aquel que se interese por un tema que en los últimos tiempos podría considerarse "de moda".

Como antes decía, creo que conozco bien qué es lo que piensa Amalia sobre la coherencia y, por ello, me parece que mis ideas al respecto -que en buena medida se han ido forjando al hilo de la lectura y la discusión de los borradores que ella iba confeccionando durante la elaboración de su trabajo-, no son muy diferentes a las suyas. Pero sí puede haber entre nosotros una diferencia de actitud que, por lo demás, explica también en una buena medida el sentido de los comentarios a sus "Diez tesis acerca de la coherencia en el Derecho" que se publican en este número de Discusiones, a cargo de Juliano Maranhâo, Claudio Michelon, Guillermo Lariguet, Aldo Schiavello y Cristina Redondo. Me refiero a lo que quizás podría llamarse "el síndrome de enamoramiento" con el tema de trabajo que, de manera explicable, afecta ("padece" sería una expresión excesiva) a quien ha empleado mucho tiempo y esfuerzo en la elaboración de una tesis de doctorado o de algún otro trabajo de investigación en el que se pone un gran empeño. En el caso de Amalia, ello se ha traducido en una disposición a salvar a las teorías de la coherencia de todas las críticas que se les han ido formulando en los últimos tiempos. Pero no lo ha hecho suscribiendo in toto una de las diversas teorías de la coherencia disponibles, sino elaborando algo así como una "super-teoría", a base de acudir a alguna de las anteriores teorías "sectoriales" (que ella conoce muy bien) para ir salvando los diversos inconvenientes que cada una de ellas podría plantear por separado. Digamos que la estrategia utilizada por Amalia es la de asumir las críticas (cuando considera que son razonables), pero sin salirse del 
paradigma de la coherencia. El resultado, como acabo de decir, es una especie de "super-teoría" que, a pesar de sus innegables méritos, puede resultar insatisfactoria, bien porque se piense que la solución de esos problemas exigiría ir más allá de una teoría de la coherencia o bien porque se considere que al ampliar tanto el ámbito de la teoría, se incurre inevitablemente en ambigüedad. En mi opinión, esto es básicamente lo que explica el sentido de los comentarios (por lo demás -y por razones obvias- ampliamente elogiosos) al texto de Amaya que se pueden encontrar en este número de Discusiones. Tanto en el caso de quienes se consideran muy próximos a su concepción filosófica, como en el de quienes muestran una mayor distancia.

Entre los primeros, Juliano S.A. Maranhâo sólo parece discrepar con el planteamiento de Amaya en la presentación lógica de los modelos coherentistas y en la defensa de una actitud más "conservadora" con respecto a la determinación de la base del sistema (para llevar a cabo las inferencias que solucionen los casos nuevos o los casos "anormales"); pero dado que el propio Maranhâo no deja de tener ciertas dudas sobre esto último (sobre si realmente discrepa o no del planteamiento de Amaya), su "objeción" podría interpretarse en el sentido de una petición de mayor precisión para evitar una ambigüedad en el texto de aquélla. En el caso de Claudio Michelon, sus discrepancias se refieren al valor y a la insuficiencia de la coherencia; pero sus diferencias radican simplemente -si yo le he entendido bien- en la necesidad de agregar a los esquemas de Amaya valores morales sustantivos, y en la necesidad de acudir también a criterios de racionalidad que van más allá de la coherencia. Y Guillermo Lariguet centra su comentario en el riesgo que ve en las teorías de la coherencia de dejar fuera el conflicto, lo que le lleva también a reclamar algo más que coherencia: "a lo mejor tenemos que rescatar cierta pizca, cierto discreto encanto, leve, muy suave, de incoherencia, que nos permita tener sensibilidad al conflicto, al disenso, a aquellos "inputs" que nuestros sistemas no recogieron y que sería relevante considerar".

En el caso de Aldo Schiavello y de Cristina Redondo, las discrepancias son algo mayores, debido seguramente a que los dos son más escépticos que Amaya en cuanto al alcance de la razón práctica, entendida como razón sobre fines y, por ello, también en relación con el 
objetivismo ético que Amalia sostiene. Pero, por lo demás, sus objeciones podrían entenderse también en términos de ambigüedad. En relación con Schiavello, lo que él encuentra insatisfactorio a propósito de la aspiración a la mejor explicación es que el esquema de razonamiento propuesto por Amaya no permite ver que la dificultad, en realidad, se halla en las elecciones valorativas que es preciso efectuar como cuestión, digamos, previa al uso de esos esquemas; y en cuanto a la conexión entre coherencia y responsabilidad epistémica, el problema radica en la ambigüedad que aqueja a este último concepto: o se interpreta la responsabilidad epistémica en un sentido tan vago que sólo puede cumplir una función ideológica, o bien presupone el objetivismo metaético y entonces -opina Schiavello- la coherencia es algo que se construye y no que se encuentra. Y en cuanto a Cristina Redondo, la conocida ambigüedad proceso-producto y la menos conocida idealempírico (la expresión "decisión" puede referirse a un contenido proposicional o a un estado de cosas del mundo) estaría, en su opinión, en la base del equívoco en que incurriría Amaya al proponer su modelo inferencial coherentista. Pues Redondo opina (y yo creo que lleva razón) que la inferencia a la mejor explicación no compite con los esquemas de la lógica deductiva (ni con los de la lógica inductiva), porque la lógica se ocupa exclusivamente de contenidos proposicionales y de resultados, y no de la acción de justificar o de decidir. Además, Cristina Redondo señala que existe una cierta ambigüedad en cuanto a si la noción de coherencia se refiere a una relación horizontal (simétrica: entre proposiciones que se apoyan mutuamente) o a una vertical (asimétrica: entre una o más proposiciones y aquellas en que las primeras se apoyan), ambigüedad que afecta a la cuestión de ¿con respecto a qué se dice que un conjunto de proposiciones o una hipótesis es coherente? Y, en fin, también ella considera que necesitamos algo más que coherencia en la justificación del razonamiento jurídico: "si no agregamos a la coherencia algo más, dado el carácter pluralista y potencialmente conflictivo de los derechos contemporáneos, un método coherentista por sí sólo no sirve para determinar una respuesta correcta" (lo que, por supuesto, no quiere decir que Cristina Redondo defienda la tesis de la respuesta correcta).

Decía antes que mis diferencias con respecto a Amalia en materia de coherencia consisten fundamentalmente en una cuestión de 
actitud. Lo que quiero expresar con ello (y en esto me parece que coincido con todos o con la mayoría de sus otros comentaristas) es que el concepto de coherencia no se puede -no se debería- ampliar tanto como para hacerlo coincidir prácticamente con el de racionalidad. Por ejemplo, a propósito de la justificación de las decisiones judiciales, yo creo que es mejor ver la coherencia (normativa o narrativa) como uno de los criterios que utilizamos para evaluar esos argumentos, pero no como el único; para estar justificada, una decisión judicial no tiene sólo que resultar "coherente", sino que cumplir también determinados requisitos lógicos, no producir (en el mundo social) consecuencias indeseables, etc. Alguien podría decir que la reducción de la racionalidad a coherencia tiene la ventaja de que facilita la construcción de una concepción unitaria de racionalidad. Pero la ventaja puede ser simplemente ilusoria si, para ello, se necesita construir un concepto tan maleable de coherencia que este último pierda -por así decirlo- sus señas de identidad. Y además hay algunos inconvenientes que derivan básicamente de que la construcción de una noción tan amplia ofrezca una respuesta a los problemas a los que trata de contestar que sea más aparente que real. Dicho de otra manera, el riesgo es el de construir un concepto de coherencia impreciso por su excesiva amplitud y que se presta, en consecuencia, a un uso ideológico. Como hablo simplemente de "riesgo", planteo ahora algunas cuestiones que me suscitan las diez tesis de Amalia y para las que no veo una fácil o satisfactoria respuesta a partir de sus planteamientos. No hace falta que aclare que el hecho de que no la vea no significa que no exista. En todo caso, ahí van mis preguntas a propósito de sus diez tesis:

1. El concepto de coherencia en el Derecho se puede entender en términos de satisfacción de restricciones.

Dejando a un lado que la concepción de Thagard quizás sea demasiado abstracta como para que pueda resultar operativa en el Derecho, ¿a qué dimensión del Derecho o de la argumentación jurídica se aplicaría esa concepción? Amalia lo hace a la argumentación judicial en materia de interpretación y de prueba, pero ¿serviría también para la argumentación legislativa? ¿No tendría más sentido decir que cuando se trata de justificar el establecimiento de una nueva ley, la coherencia opera más bien como un límite, pero que no tiene el papel rector que 
cumple en la argumentación judicial? ¿Valdría la misma noción de coherencia cuando ese concepto se refiere a decisiones que cuando lo hace a los elementos de un sistema jurídico (se trataría de la coherencia como relación vertical u horizontal a la que se refería Cristina Redondo)?

2. La inferencia coherentista es una inferencia de tipo explicativo.

¿Pero por qué contraponer la inferencia coherentista -la abducción- a la deducción y a la inducción? ¿No se trata realmente de un malentendido, puesto que se habla de "inferencia" en sentidos distintos? El hecho de que Amalia hable de tres etapas (descubrir los elementos relevantes; elaborar o refinar las alternativas de decisión que resultan, en principio, plausibles; evaluar y comparar las alternativas para seleccionar una), ¿no es acaso señal de que Cristina Redondo tiene razón cuando señala que la inferencia coherentista se refiere a una actividad, a un proceso? ¿Hay realmente algo de novedoso en la abducción que no pueda explicarse en términos de una inducción realizada en el contexto de descubrimiento y que, por tanto, tiene un carácter derrotable? Si esto fuera así, ¿no parece exagerada la afirmación de que "la coherencia no puede formalizarse con las herramientas lógicas tradicionales"? ¿Acaso necesitamos acudir a la abducción si de lo que queremos dar cuenta es de la motivación producida por un órgano judicial? Y si lo que quisiéramos formalizar fuera el proceso, la actividad argumentativa que tiene lugar, por ejemplo, en el interior de un tribunal (no el texto de la sentencia), ¿sería suficiente con la "inferencia a la mejor explicación”? ¿No necesitaríamos esquemas dialécticos de mucha mayor complejidad?

3. La coherencia se construye mediante operaciones de contracción, adición y reinterpretación.

Si el tercero de los mecanismos, la reinterpretación, fuera distinto de los otros dos (no consistiera en eliminar o en añadir algo -algún significado posible de los enunciados del sistema-), ¿cabría seguir hablando de coherencia? ¿Se podría innovar el sistema de esa manera -digamos, de una manera radical- en nombre de la coherencia? Por otro lado, Amalia considera que, a diferencia de la operación de 
contracción, la de adición es "menos conocida” en el Derecho. ¿Pero no consiste en eso la analogía en sentido amplio (que, en mi opinión incluiría tanto el argumento a pari como los argumentos a contrario y a fortiori), esto es, en añadir -si se trata por ejemplo de un argumento a pari- a las premisas de partida un enunciado de principio -que podría considerarse implícito- de que los casos esencialmente semejantes deben recibir el mismo trato y otro enunciado que afirma que el nuevo caso es esencialmente semejante a uno anterior que ha sido resuelto de una determinada manera? ¿Implica lo que Amalia está aquí sosteniendo algo distinto a afirmar que los argumentos interpretativos característicos del Derecho (además del modus ponens) son la analogía (en el sentido amplio antes indicado) y la reducción al absurdo (que se emplea para eliminar un significado posible de una norma o una de las dos posibles hipótesis fácticas)?

4. La responsabilidad epistémica es un componente central en una teoría de la coherencia.

¿Pero no cabría decir lo mismo de cualquier otra teoría? Por ejemplo, alguien que suscriba una concepción de la verdad como correspondencia, ¿acaso no aceptaría también el "deber de creer de acuerdo con las pruebas o el de buscar pruebas acerca de proposiciones cuyo valor de verdad es incierto a la luz de las pruebas disponibles"? ¿Y no estaría de acuerdo en considerar que una conducta epistémicamente responsable es aquella que resulta "del ejercicio de una serie de virtudes (...) tales como la diligencia, la valentía para enfrentar la crítica, la perseverancia o la apertura de mente”? ¿Se necesita, en definitiva, ser coherentista para adherirse a un código de ética judicial que, como ocurre con el Código Modelo Iberoamericano, incorpora tanto deberes deónticos como virtudes judiciales?

5. Los estándares coherentistas de la justificación jurídica varían con el contexto.

De nuevo: ¿acaso es característico de una concepción coherentista de la justificación jurídica ser sensible al contexto? ¿No es el contexto uno de los criterios más tradicionales de interpretación 
jurídica, un mecanismo usual de deshacer ambigüedades, etc.? ¿A qué otros estándares se enfrentan en este sentido los de naturaleza coherentista? ¿Cuál es la teoría de la justificación jurídica -salvo alguna crudamente formalista que no creo haya muchos que defiendan- que se opone a tomar en consideración el contexto?

\section{El razonamiento coherentista es un razonamiento acerca de fines.}

Dejando a un lado que la tesis de Amalia está formulada con cierta ambigüedad que quizás explica una de las críticas de Schiavello (sobre si el razonamiento coherentista es o no compatible con una concepción instrumental de la razón práctica), ¿es razonable pensar que la deliberación sobre fines está exclusivamente -o esencialmente- guiada por alguna idea de coherencia? Yo no dudo de que la coherencia juega aquí un papel, pero para deliberar sobre fines antes hay que determinar cuáles son esos fines y no veo que eso sea función de una teoría de la coherencia: ¿o también habría que considerar coherentista, por ejemplo, el método de Kant para llegar a las tres formulaciones del imperativo categorico? ¿Es realmente distinto el método del especificacionismo o el de las razones pro tanto de Hurley en relación con el de la ponderación? ¿En qué radica la superioridad de los dos primeros sobre el segundo? ¿Y qué pasa, por cierto, en relación con los casos trágicos que tanto preocupan a Lariguet? ¿Tiene la teoría (o alguna teoría) de la coherencia una solución para ellos? ¿Cómo es posible que una teoría puramente coherentista (que no incorpore criterios de racionalidad o de justificación distintos al de la coherencia) no asuma "una visión no conflictual del Derecho"? ¿Acaso no es la coherencia precisamente lo contrario del conflicto?

7. Hay tres tipos de razones a favor de la coherencia: epistémicas, prácticas y constitutivas.

Esas razones justifican, sin duda, que se atribuya una gran importancia a la coherencia en el Derecho, ¿pero son suficientes como para convertir a la coherencia en el único o incluso el principal criterio cuando se trata de evaluar las argumentaciones sobre cuestiones de hecho o sobre cuestiones normativas? Si las "creencias acerca del 
Derecho justificadas en virtud de su coherencia son, probablemente, creencias verdaderas o correctas", ¿no significa eso que necesitamos de alguna otra teoría - una teoría no coherentista- acerca de la verdad o de la corrección?

8. La motivación principal de las teorías coherentistas es la articulación de una alternativa no escéptica al formalismo.

¿Pero no podría decirse que el intento de encontrar una vía media entre el formalismo y el escepticismo es una característica de todas las teorías de la racionalidad práctica, esto es, de todos aquellos que sostienen una concepción amplia de la racionalidad? Sin ir más lejos, ¿no era esa la pretensión de Perelman y de todos los autores que integran lo que se suele llamar "teoría estándar de la argumentación jurídica"? ¿Habría que considerar también como coherentistas a todas estas teorías? ¿No se estará identificando demasiado rápidamente coherencia con racionalidad o con racionalidad práctica? Por cierto, ¿qué es lo que se está entendiendo aquí por "positivismo formal”? ¿Cabría incluir también en él una concepción como la de Hart? ¿Es lo mismo "formal" que "formalista"?

9. El coherentismo es una teoría plausible desde un punto de vista psicológico y ésta es una buena razón a favor de la misma.

Es cierto que en la medida en que el modelo coherentista no se aleje mucho de la práctica argumentativa, eso facilita que pueda contribuir a la mejora de esa práctica, en el sentido de que quienes participan en la misma pueden estar más dispuestos a aceptar la coherencia como criterio de justificación. ¿Pero no supone eso un riesgo de conservadurismo y, lo que es peor, una llamada -como la propia Amalia reconoce- a "fabricar" coherencia donde realmente no existe? Ella considera que el remedio para ese problema está en una teoría de la "responsabilidad epistémica" que, por lo demás, formaría parte de esa "super-teoría" de la coherencia a la que antes me refería, ¿pero no suena esto a demasiado circular? Y, por lo demás, ¿no supone de nuevo volver a las cuestiones planteadas en el anterior punto 4 ? 
10. El coherentismo sitúa al agente en el centro de una teoría de la justificación.

¿Pero no podría decirse lo mismo de muchas otras teorías de la justificación que parecería abusivo considerar "coherentistas"? ¿Acaso hay que ser coherentista para poder hablar de "virtudes judiciales" o del ideal del "buen juez"? Y, por cierto, a la vista del entusiasmo que Amalia parece sentir por las teorías "aretaicas" (al hablar de "responsabilidad epistémica" señala que mientras que antes era partidaria de una concepción ecléctica, que combinara los deberes epistémicos con las virtudes, ahora parece optar más bien por una concepción exclusivamente "aretaica"), ¿no estaremos ante un nuevo "síndrome de enamoramiento" con respecto al tema de investigación que en el caso de Amalia ha seguido al de la coherencia? ¿No hay una cierta exageración al afirmar que la ética jurídica no es "una disciplina independiente de la teoría de la argumentación jurídica, sino una parte esencial de la misma"? ¿No existe también aquí el riesgo de confundir una buena argumentación judicial con la argumentación llevada a cabo por un buen juez? ¿No conviene ser cautelosos frente a cualquier intento de identificar la ética judicial con la doctrina de las virtudes judiciales?

No pretendo, naturalmente, que Amalia conteste a todas las anteriores cuestiones (que -insisto- coinciden en buena medida con las observaciones que sus comentaristas le hacen en este número de Discusiones). Y no sólo por razones de espacio, sino porque ella podría considerar que muchas de esas objeciones ya están planteadas y contestadas en su libro (del cual, estas "Diez tesis" no son más que un apretado resumen). Pero lo que yo quiero señalar con lo anterior -lo recuerdo una vez más- es simplemente la inconveniencia de ampliar tanto el concepto de coherencia que el mismo pase a coincidir con el de racionalidad. No se trata, pues, de una objeción sustantiva, sino más bien de carácter metodológico. Por lo demás, y puestos a ser virtuosos, creo que es de justicia que acabe mi comentario mostrando mi admiración y agradecimiento por su artículo y por su libro. No creo que en los últimos tiempos se hayan producido muchos textos iusfilosóficos de los que el lector pueda aprender tanto. 